\title{
Traducir las escrituras desterritorializadas: del elogio de la creolidad del autor al elogio de la marginalidad del traductor
}

\author{
María Eugenia Ghirimoldi \\ Universidad Nacional de La Plata \\ ghirimoldi.mae@gmail.com
}

\begin{abstract}
This paper offers an analysis of heterolingualism and translation in La grande drive des esprits by Gisèle Pineau (1993) and its Spanish translation, Una antigua maldición (1999). Based on the literary theories around Creoleness and on the theories of translation centered on a "new translation ethics", it analyzes heteroligualism and "writing-translation" in Pineau's work looking at the various mechanisms deployed in its Spanish translation, which privileges linguistic creativity. The paper has a dual goal: (1) to characterize the language of Pineau's work as deterritorialized in order to identify the ways in which the creole language is inserted in it and allows the author, along the lines of the creolists' ethical and aesthetic proposals, to offer a language of resistance against hegemonic colonial language uses; and (2) to examine the translation techniques employed and bring forth theoretical adaptations fitting to analyze this type of translation. The paper concludes with a reflection of the translator's possibilities to retain or "recreate" (marginalize?) the heterolingualism of the original work.
\end{abstract}

Key words: heterolingualism; writing-translation; creole; linguistic recreation; marginalization

Résumé : À partir du roman La grande drive des esprits de Gisèle Pineau (1993) et de sa version en espagnol, Una antigua maldición (1999), et recourant aux postulats théoriques littéraires de la créolité et aux théories de la traduction de la " nouvelle éthique de la traduction », nous étudions l'hétérolinguisme en « écriture-traduction » et les différents mécanismes appliqués à la traduction vers l'espagnol où la créativité linguistique occupe un espace privilegié. Notre objectif est double : (1) caractériser la langue d'écriture déterritorialisée pour identifier les modes d'insertion de la langue créole qui permettent de construire une langue de résistance contre l'utilisation hégémonique de la langue coloniale, en accord avec les postulats éthiques et esthétiques des créolistes; (2) examiner les techniques de traduction et ajuster les modèles pour l'analyse des traductions. Nous proposons une réflexion sur le fait que le traducteur puisse conserver ou « recréer » (marginaliser?) l'hétérolinguisme original.

Mots clés : hétérolinguisme; écriture-traduction; créole; recréation linguistique; marginalité

Resumen: A partir de La grande drive des esprits de Gisèle Pineau (1993) y su versión al español, Una antigua maldición (1999), desde los postulados teóricos literarios de la Creolidad y las teorías de la traducción de la "nueva ética traductora", analizamos el heterolingüismo en la "escritura-traducción" y los diferentes mecanismos aplicados en su traducción al español donde la creatividad lingüística ocupa un lugar de privilegio. Nuestro objetivo es doble: (1) caracterizar la lengua de escritura desterritorializada para identificar los modos de inserción de la lengua creole que permiten construir una lengua de resistencia contra el uso hegemónico de la lengua colonial, en consonancia con los postulados éticos y estéticos de los creolistas; (2) examinar las técnicas de traducción y ajustar los modelos para el análisis de las traducciones. Ofrecemos nuestra reflexión sobre las posibilidades que el traductor o la traductora tiene en sus manos de conservar o "recrear" (¿marginalizar?) el heterolingüismo original.

Palabras clave: heterolingüismo; escritura-traducción; creole; recreación lingüística; marginalidad 
Resumo: Com base na obra La grande drive des esprits (1993), de Gisèle Pineau, e sua versão em espanhol, Una antigua maldición (1999), nos postulados teóricos literários da crioulidade e nas teorias da tradução da "nova ética tradutória", analisamos o heterolinguismo na "escritura-tradução" e os diferentes mecanismos aplicados na tradução da obra para o espanhol, onde a criatividade linguística ocupa lugar privilegiado. Nosso objetivo é duplo: (1) caracterizar a língua de escritura desterritorializada a fim de identificar os modos de inserção da língua crioula que nos permitem construir uma língua de resistência contra o uso hegemônico da língua colonial, de acordo com os postulados éticos e estéticos dos crioulistas; (2) examinar as técnicas de tradução e ajustar os modelos para a análise das traduções. Apresentamos nossa reflexão sobre as possibilidades que o tradutor tem em mãos de preservar ou "recriar" (marginalizar?) o heterolinguismo original.

Palavras-chave: heterolinguismo, escritura-tradução, crioulo, recriação linguística, marginalidade

\section{Introducción}

Nos proponemos en este trabajo analizar el heterolingüismo en la escritura de Gisèle Pineau en La grande drive des esprits (1993) y su versión al español, Una antigua maldición (1999), en el marco de los postulados teóricos literarios de la Creolidad y de las teorías de la traducción de la "nueva ética traductora". Luego de presentar el contexto de enunciación socio-literario periférico en el que emerge la novela elegida, alcanzar un doble objetivo: por un lado, caracterizar la escritura desterritorializada de la autora e identificar los modos de inserción de la lengua minorizada, el creole, en el texto de partida o escritura-traducción; por otro, analizar las opciones llevadas a cabo en la traducción para reescribir el texto en un español recreado. Finalmente reflexionaremos sobre una de las técnicas para traducir la hibridez, la recreación lingüística.

Antes de lanzarnos en nuestros análisis dedicaremos unas líneas para presentar a la autora y a la obra elegida. Gisèle Pineau, quien se define como "Esclava de ayer, francófona de hoy" (Pineau, "Le Sens"), es una de las escritoras de la literatura antillana contemporánea más reconocidas en el mundo literario francófono. Entre su prolífica obra literaria y ensayística que incluye cuentos, literatura juvenil y ensayos, elegimos la primera novela de la autora, titulada La Grande Drive des Esprits, editada en París en 1993 por Le Serpent à Plumes. Contemplamos también su traducción, publicada con el título Una antigua maldición y realizada por el traductor español Manuel Serrat Crespo, editada en Barcelona en 1999 por Ediciones del Bronce. Por la novela recibirá el Premio de lectoras de Elle 1994 y el Carbet del Caribe 1993.

Hija de la diáspora, nacida en París en 1956, de padres guadalupeños, Pineau afirma haber comenzado a escribir a los 7 años cumpliendo con su vocación literaria que le permite luchar, en su triple condición de mujer, negra y creole, contra el racismo y la violencia.

Según sus propias palabras, el ingreso a la escritura a temprana edad se le presentó al igual que lo hizo su abuela que la acompaño durante sus dolorosos años en Francia: 
Ella (la escritura) me rescató del aislamiento, de los tormentos de la búsqueda, me acompañó en mis miedos y mis llantos, me abrió horizontes insospechados, la libertad de expresión, el poder la creación. ${ }^{1}$ (Pineau, "L'identité" 220)

\section{Fundamentos teóricos: El elogio de la creolidad por parte de la autora}

Recuperando el imaginario antillano y dando voz a sus ancestros silenciados, la escritura de Pineau entra en consonancia con sus colegas martiniqueños Jean Bernabé, Raphaël Confiant y Patrick Chamoiseau, autores del manifesto literario de la creolidad. Los autores del Elogio de la Creolidad (1993) continúan los esfuerzos iniciados en la búsqueda de identidad por sus antecesores defensores, en un primer momento, de la Negritud, que volvía la mirada hacia África para revalorizar el legado cultural afro-caribeño, liderados por Aimé Césaire (cuya obra de referencia es el Cahier d'un retour au pays natal de 1939), y en un segundo momento, de la antillanidad postulada por Êdouard Glissant (condensada en su obra Le discours antillais de 1981), que proponía recuperar la especificidad antillana a partir de la búsqueda no ya de una raíz única anclada en un territorio sino como una convergencia submarina de raíces ramificadas (Glissant 128).

En el Elogio los autores precisan cinco aspectos que caracterizan la expresión literaria de la Creolidad: 1) el enraizamiento en la tradición oral, 2) la puesta al día de la memoria verdadera expresada por el pueblo y silenciada por el discurso occidental, 3) la temática de la existencia en donde no existe nada que sea pequeño del imaginario local, 4) la irrupción de la modernidad en la búsqueda de una estética nueva sin la cual no hay arte.

Esos primeros cuatro aspectos se pueden percibir en la historia contada en $\mathrm{La}$ grande drive des esprits. Léonce, un joven negro trabajador incansable, se gana el amor de la bella Myrtha con perseverancia, a pesar de su deformidad de nacimiento, un pie torcido, considerado un don sobrenatural que puede atraer a los malos espíritus. Cuando todo parece tomar un rumbo de prosperidad y armonía en la vida del matrimonio y sus hijos, comienzan a caer las maldiciones que se traen de generación en generación sobre la familia. A través del relato de la narradora principal la autora introduce los temas de las creencias religiosas, la superstición, la castidad, el acoso sexual, la sumisión de la mujer, el duelo, la tradición oral, el feminismo. La obra explota el imaginario antillano de lo sobrenatural con una escritura que conmueve, emociona, moviliza el lector, donde el dolor y el sufrimiento están omnipresentes como herencia indeleble del pasado esclavista.

El quinto aspecto sobre el que nos detendremos para nuestro análisis es 5) la elección de la palabra. Para los creolistas, el creole, la lengua primera, "es el vehículo original del yo profundo de los martiniqueños, guadalupeños, guayaneses, mascareños, la lengua con la cual sueñan y la que les provee la fuerza de su expresividad. Pretender renunciar al creole significa un suicidio estético, una amputación cultural" (Bernabé, Confiant y Chamoiseau, Elogio 36-42).

\footnotetext{
1 « Elle m'a sauvé de l'isolement, des tourments de la quête, m'a accompagnée dans mes peurs et dans mes pleurs, m'a ouvert des horizons insoupçonnés, la liberté d'expression, le pouvoir de la création » (Mi traducción).
} 
Este aspecto en Pineau se revela profundamente sensible, en su particular debate personal en la selección de lenguas, con un espíritu abierto a lo mútliple y lo diverso:

Abuso de las palabras que me han prestado. Francesas, creoles, inglesas, latinas, españolas, sus comarcas de origen poco importan mientras suenen como verdaderas en mi corazón y estén en armonía con la mirada que tengo sobre el mundo (...). (Pineau, "L'identité", 222) ${ }^{2}$

\section{La lengua desterritorializada de Pineau}

Como explica Pascale Casanova, cada escritor que entre en el universo literario reactualiza toda su herencia literaria nacional y lingüística, impregnando su marca personal en relación a ese legado. Para crear las condiciones de su aparición en ese espacio desde un contexto periférico, la literatura antillana, Gisèle Pineau defiende su identidad guadalupeña y creole escribiendo en una lengua desterritorializada, haciendo que la lengua del centro, su lengua materna, sea transformada y recreada con el creole, la lengua de sus ancestros. Por esta razón es posible incluir a esta autora dentro de la llamada escritura de minorías, es decir, aquella que Gilles Deleuze y Félix Guattari (28) definen como la literatura promovida por una minoría que escribe en una lengua mayor. Uno de los rasgos que la caracterizan es la desterritorialización de la lengua mayoritaria, es decir, esta lengua —el francés en este caso—pierde "territorio" al ser socavada por la lengua minoritaria que desestabiliza su estatus de lengua dominante. La lengua aquí, en el caso de escritores de espacios descentrados, constituye el objeto primordial de las luchas por afirmar sus diferencias, el único material de creación y de resistencia.

Desde una perspectiva poscolonial el fenómeno plurilingüe se convierte en objeto de estudio destacado. En la obra de referencia sobre las técnicas de la escritura plurilingüe The Empire Writes Back, publicada en 1989, Bill Ashcroft, Gareth Griffith y Helen Tiffin definen dos técnicas empleadas en la literatura poscolonial: la abrogación o rechazo del privilegio del "inglés" y la apropiación o reconstitución de la lengua del centro que es remodelada para nuevos usos. Ambas técnicas se complementan y cada una imprime su sello distintivo a las literaturas poscoloniales que construyen su espacio entre culturas (Ashcroft et al. 39). Estos procedimientos son percibidos y nombrados de diferentes maneras. Chantal Zabus en 1991 denomina palimpsesto a las técnicas de "indigenización" de la lengua europea que emplean los escritores africanos. Según su visión, el hipertexto francés deja entrever el hipotexto de las lenguas africanas (Zabus 2-3). Por su parte Patrick Scott, en sus estudios sobre la obra The Voice del novelista nigeriano Gabriel Okara propone el término "translingüismo" que define como la reproducción intencional y artística de los rasgos de una lengua que se hacen presentes en otra (Scott 75). Este fenómeno de hibridación lingüística, desde la perspectiva textual, ha sido designado por Rainier Grutman como heterolingüismo: "la

\footnotetext{
2 « J'abuse des mots qui me sont prêtés. Français, créoles, anglais, latins, espagnols, leurs contrées d'origine importent peu dès lors qu'ils sonnent vrai, selon mon cœur, et sont en concordance avec le regard que je porte sur le monde » (Mi traducción).
} 
presencia dentro de un texto de idiomas extranjeros, en cualquiera de sus formas o variedades (sociales, regionales o cronológicas) de la lengua principal" (37).

\section{El texto de partida como escritura-traducción}

Los textos heterolingües desestabilizan el paradigma tradicional del monolingüismo que puede encontrar entre sus causas la ideología romántica basada en la correspondencia ideal entre una lengua, una literatura y una nación. Estas relaciones no se sostienen en las escrituras poscoloniales donde la práctica plurilingüe se vuelve la norma y la heterogeneidad lingüística es parte constitutiva de su fuerza creadora. Las escrituras poscoloniales ponen en entredicho la oposición tradicional entre original y traducción, desde el momento en que vehiculan sentidos en más de una lengua. Una de las primeras escritoras en observar este hecho en el mundo francófono fue Samia Mehrez en su estudio de la literatura francófona africana, donde destaca la presencia de un lenguaje mestizo cuyo fin es doble: descolonizarse del opresor y recuperar la propia cultura marginalizada. Esta idea es retomada por María Tymoczko ("Post-colonial Writing", "Translation of Themselves") para desarrollar su teoría de la escritura poscolonial como traducción literaria interlingüe donde se trasponen aspectos de la lengua nativa, patrones culturales, creencias y tradiciones. Para la crítica estadounidense estas escrituras poseen una carga referencial doble que comunica de modo diferencial a los lectores según el grado de familiaridad con la cultura minorizada que subyace. Esto constituye un medio privilegiado para los sujetos ex-colonizados de resistir y rebelarse contra la dominación.

\section{Modo de inscripción del creole en la novela antillana}

A partir de los años noventa los novelistas antillanos rediseñan el mito de la creolidad a través de la escritura " inventada " por uno de sus precursores, Patrick Chamoiseau, que funda un estilo " chamoisizado », como lo bautizara el checo Milan Kundera (58), de inseminación del créole en la lengua francesa, especialmente en su novela Texaco, de 1992. Entre estos autores que escriben desde la creolidad se destacan Raphael Confiant, Ernest Pépin y Gisèle Pineau. La literatura francófona de las Antillas a partir de entonces se escribe en un francés regional o creolizado, donde por medio de diversos mecanismos, el creole se hace presente a modo de préstamos y frases insertadas o bien se representa ${ }^{3}$ en la lengua francesa, invadiéndola, deformándola, enriqueciéndola, en todos los niveles, léxico, semántico, sintáctico, morfológico o fonológico. Diferenciamos aquí a grandes rasgos dos tipos de integración del creole: unidades intermedias $u$ oraciones y unidades mínimas que no pueden dividirse en unidades menores sin perder el sentido. Estas últimas abarcan fenómenos lingüísticos que involucran a más de un componente de la lengua, para cuyo análisis resulta pertinente el modelo de interfaces utilizado por Céline Desmet Argain para su estudio sobre el translingüismo en Texaco de Chamoiseau (1992). Por su parte, Pascale De Souza, en su estudio sobre textos francófonos, define diversas formas de integración

\footnotetext{
${ }^{3}$ El destacado es nuestro.
} 
del creole que van desde las citas, la integración parcial del creole en el francés, hasta llegar a la creolización del francés.

Cada autor o autora hace un uso particular de la lengua francesa creolizada. Pineau imprime su marca a esta hibridez lingüística y se diferencia de Chamoiseau y de Confiant en el sentido de que ella ofrece menos autotraducciones y explicitaciones que sus predecesores, prefiriendo un estilo donde la lengua minorizada aparece ocupando el mismo nivel que la lengua dominante, sin marcas ni explicaciones, en una armoniosa cohabitación. En efecto, según el estudio de Marie-Christine HazaëlMassieux sobre el contexto de utilización del creole y las representaciones vehiculizadas por las novelas antillanas francófonas, cuando Chamoiseau y Confiant recurren al creole, en el discurso directo o indirecto y en algunas citas, se acompañan de traducciones a continuación, al pie de página o entre paréntesis. Su uso del creole caracteriza emblemáticamente la pertenencia social y la emoción que afecta al personaje que lo pronuncia. Es sabido que según la manera de hablar de cada persona es posible clasificarla a nivel individual y social. En un país creolófono donde la lengua dominante es el francés, alguien que hablara en creole, hasta no hace mucho tiempo, podía ser ligado a un estrato social bajo, con poca educación, y hasta a ideas de vulgaridad (Bernabé 142). Este prejuicio lo podemos trasladar a la obra literaria, donde los personajes se manifiestan con un determinado lenguaje. En una novela en creole de Confiant, por ejemplo en Marisosé (1987), los personajes que hablan en creole son aquellos que se supone hablarán en esa lengua, en cambio aquellos que asumen un papel de francófonos monolingües, toman la palabra en francés.

Es preciso destacar, como advierte Hazaël-Massieux, en relación con la extensión de las novelas, que estas ocurrencias existen en un número reducido, como lo indican los datos siguientes que recogimos de su trabajo y a los que sumamos los nuestros sobre Pineau.

\section{Tabla 1}

Comparación de cantidad de oraciones en creole, tipo y función que cumplen dentro de la situación en que son insertadas, en Chamoiseau, Confiant y Pineau, dentro de una cantidad de páginas equiparable en las novelas seleccionadas.

\begin{tabular}{|l|l|l|l|l|}
\hline Novela (Autor, Año) & $\begin{array}{l}\text { Pág. } \\
\text { No- } \\
\text { vela }\end{array}$ & $\begin{array}{l}\text { Cant. } \\
\text { Orac. en } \\
\text { créole }\end{array}$ & $\begin{array}{l}\text { Tipo de frase } \\
\text { dominante }\end{array}$ & Función \\
\hline $\begin{array}{l}\text { Chronique des Sept Misères } \\
\text { (Chamoiseau, 1986) }\end{array}$ & 240 & 31 & Exclamativas & Enojo/insulto \\
\hline Chemin d'école (Chamoiseau, 1994) & 202 & 10 & $\begin{array}{l}\text { Interrogativas y } \\
\text { exclamativas }\end{array}$ & Discusión \\
\hline L'allée des soupirs (Confiant, 1994) & 200 & 72 & Exclamativas & $\begin{array}{l}\text { Discusión/ } \\
\text { Agresión }\end{array}$ \\
\hline $\begin{array}{l}\text { La grande drive des esprits (Pineau, } \\
\text { 1993) }\end{array}$ & 210 & 18 & Exclamativas & $\begin{array}{l}\text { Consejos/ } \\
\text { ruegos/ etc. }\end{array}$ \\
\hline
\end{tabular}

Fuente: Elaboración propia

\footnotetext{
${ }^{4}$ El cuadro es nuestro.
} 
La tabla 1 muestra para cada una de las obras observadas el número total de páginas, la cantidad de oraciones en creole insertadas, el tipo de frase que predomina (exclamativas e interrogativas) y la función que cumplen dentro del contexto de situación (expresión del enojo, del insulto, de la discusión, de los consejos, de los ruegos, etc.).

Como ilustran estos datos, Confiant se destaca por su mayor uso del creole debido, por un lado, a su fenomenal dominio de la lengua (es el único entre los autores arriba citados que ha escrito tres novelas enteramente en creole, además de un diccionario) y, por otro lado, a que sus personajes pertenecen a las clases más bajas de la sociedad, a las que se les niega el acceso a la lengua francesa. Cuantitativamente, Pineau se posiciona en un nivel promedio comparable a Chamoiseau en términos de relación cantidad de páginas / cantidad de oraciones insertadas. En cuanto a los tipos de frase, los cuatro autores comparten el uso principal de exclamativas tomadas dentro de diálogos directos o discursos referidos, con algunas citas de proverbios y canciones, como veremos en detalle en nuestra autora elegida. Lo que distingue a Chamoiseau y a Confiant de Pineau es el tipo de función que cumple el uso del creole. Los primeros se caracterizan, como afirma HazaëlMassieux, por expresar sentimientos de conflicto y agresión, en boca de personajes marginados. En Pineau, en cambio, se hace un uso más diversificado y de connotación más positiva, ofreciendo una representación de la lengua creole que refleja los sentimientos más íntimos del ser humano.

\section{Inserción de oraciones en creole en La grande drive des esprits}

Encontramos en la novela elegida 18 ocurrencias de las cuales 17 fueron frases exclamativas y una corresponde a la inserción de una canción (tabla 1). En un volumen de 210 páginas no parece demasiado pero resulta suficiente como para causar un efecto de extrañamiento y para que despierte la curiosidad en el lector por esta lengua dominada. El heterolingüismo no está marcado por cambio de tipografía, una estrategia de escritura que, ante la ausencia de "balizas", sorprenden aún más al lector francófono sea creolófono o no. En toda la obra no se registra ningún cambio tipográfico entre el francés y el creole, en tanto lenguas "indígenas" según el término de Jean Bernabé (Arsaye 146) y siendo el único caso de uso de cursiva el uso de préstamos del latín, en tanto lengua "exógena".

En todos los casos se trata, como dijimos más arriba, de oraciones exclamativas que reflejan el discurso oral cargado de alguna emoción. Se trata exclusivamente de enunciados en estilo directo que dan voz a distintos personajes, todos habitantes de las zonas rurales de la Gran Tierra, entre los que se encuentra la narradora, quien había pasado tres años estudiando en París luego de obtener su bachillerato y también hace uso del creole en dos circunstancias. Los dos personajes que no emiten ninguna oración en creole son Léonce y Myrtha. En cambio, quienes utilizan estas expresiones hemos identificado a: gente del barrio, Sosthène , Barnabé , Ninette , Octavie , Agathe , Paul y la narradora. Estas exclamaciones reflejan una variedad de sentimientos y situaciones como fastidio, aliento, tristeza y ternura en la canción de cuna, predicción, advertencia, consejo, plegaria y dichos proverbiales. La extensión de las frases llega a 17 palabras como máximo, considerando la inserción de la canción aparte como 
citación. A continuación mostramos los pasajes extraídos, en los que, para facilitar la observación, destacamos en negrita los términos en creole (indicamos entre paréntesis al final de cada segmento el número de página en que aparecen en las obras citadas).

\section{Tabla 2}

Muestra de los pasajes insertados en creole en la obra original, La grande drive des esprits, indicando los personajes que los emiten y la situación en que tienen lugar. En la última columna, su traducción en la versión publicada en español, Una antigua maldición.

\begin{tabular}{|c|c|c|c|c|}
\hline $\mathrm{N}^{\circ}$ & Pasaje & Personaje & Situación & Traducción Publicada \\
\hline 1 & $\begin{array}{l}\text { C'est bon! Ça peut aller! I ké } \\
\text { kenbé! Ça va tenir! (25) }\end{array}$ & $\begin{array}{l}\text { la gente à } \\
\text { Léonce }\end{array}$ & Aliento & $\begin{array}{l}\text { ¡ya está!, ipuede servir!, } \\
\text { ¡v'aguantar!, ¡resistirá! (28) }\end{array}$ \\
\hline 2 & $\begin{array}{l}\text { On ti pisa an kay fè, chè! } \\
\text { Pisser, seulement pisser, } \\
\text { Ninette! (61) }\end{array}$ & $\begin{array}{l}\text { Sosthène a } \\
\text { su mujer } \\
\text { Ninette }\end{array}$ & $\begin{array}{l}\text { Queja/ } \\
\text { fastidio }\end{array}$ & $\begin{array}{l}\text { ¡On ti pisa an kay fè, chè! } \\
\text { ¡Mear, sólo mear, Ninette! } \\
(66)\end{array}$ \\
\hline 3 & $\begin{array}{l}\text { Lè pitit an mwen/ Ka mandé } \\
\text { tété/ Mwen ka lé bay/ Manjé } \\
\text { matété/ Pitit dodo/ Papa pa } \\
\text { la/ Sé manman tou sèl/ Ki } \\
\text { dan lanbara/ Pitit dodo/ } \\
\text { Papa pa la/ Sé manman tou } \\
\text { sèl/ Ki dan lanmizè (70) }\end{array}$ & $\begin{array}{l}\text { Barnabé a la } \\
\text { narradora }\end{array}$ & $\begin{array}{l}\text { Tristeza/ } \\
\text { Ternura }\end{array}$ & $\begin{array}{l}\text { Mi nenito/Tere teta/ Ke le vi a } \\
\text { da/ Pa komel puré/ Arroró/ } \\
\text { Papa no ta/ Su mami ta sola/ } \\
\text { No tiene na/ Arroró/ Papa no } \\
\text { ta/ Su mami ta sola/ No } \\
\text { tienen reá... (75). }\end{array}$ \\
\hline 4 & $\begin{array}{l}\text { Kitèy mò! Elle va te tuer, } \\
\text { cher! Madanm-lasa sé on } \\
\text { vyé volan! Laisse-la mourir!" } \\
\text { (76) }\end{array}$ & $\begin{array}{l}\text { Ninette a su } \\
\text { hijo Léonce }\end{array}$ & Advertencia & $\begin{array}{l}\text { ¡Kitèy mò! ¡Va a matarte, } \\
\text { querido! ¡Madanm-lasa sé } \\
\text { on vyé volan! ¡Déjala morir! } \\
\text { (82) }\end{array}$ \\
\hline 5 & $\begin{array}{l}\text { Zot ké ni kat ti moun! N'en } \\
\text { demande pas davantage au } \\
\text { Seigneur! (81) }\end{array}$ & $\begin{array}{l}\text { Octavie, la } \\
\text { difunta } \\
\text { abuela } \\
\text { durante una } \\
\text { aparición a } \\
\text { Leonce } \\
\end{array}$ & Predicción & $\begin{array}{l}\text { ¡Zot ké ni kat ti moun! ¡No } \\
\text { pidas más al Señor! (88) }\end{array}$ \\
\hline 6 & $\begin{array}{l}\text { On bèl ti fi, bien belle, oui! } \\
\text { (89) }\end{array}$ & $\begin{array}{l}\text { Ninette a } \\
\text { Leonce } \\
\text { cuando nace } \\
\text { su hija y él } \\
\text { estaba } \\
\text { borracho }\end{array}$ & Halago & $\begin{array}{l}\text { ¡Una bonita nena, mu bonita, } \\
\text { sí! (96) }\end{array}$ \\
\hline 7 & Padon bondyé! (91) & $\begin{array}{l}\text { Octavie a } \\
\text { Leonce en su } \\
\text { segunda } \\
\text { aparición } \\
\text { cuando se } \\
\text { arrepiente de } \\
\text { su } \\
\text { borrachera }\end{array}$ & Reprimenda & ¡Peldón dio-mio! (98) \\
\hline 8 & Bon dié...! 97 & $\begin{array}{l}\text { Sosthène en } \\
\text { oración }\end{array}$ & Plegaria & ¡Dios mío...! 104 \\
\hline
\end{tabular}




\begin{tabular}{|c|c|c|c|c|}
\hline 9 & $\begin{array}{l}\text { Sé ti moun! Yo pas biswen } \\
\text { a yen dot ki manjé, kaka, } \\
\text { pisé, domi! ...(98) }\end{array}$ & $\begin{array}{l}\text { Agathe a su } \\
\text { suegra } \\
\text { Ninette }\end{array}$ & Tranquilidad & $\begin{array}{l}\text { ¡To chavale! ¡Tos comé, } \\
\text { cagé, meá, dolmí! (105) }\end{array}$ \\
\hline 10 & $\begin{array}{l}\text { Bon dié, ban mwen kouraj! } \\
\text { (107) }\end{array}$ & $\begin{array}{l}\text { Ninette en } \\
\text { oración }\end{array}$ & Plegaria & ¡Dame való, dio mío! (113) \\
\hline 11 & $\begin{array}{l}\text { Lapenn ki mété-y la! } \\
\text { Lapenn pwan-y davwa yo } \\
\text { pa té vlé-y, pa rapot a vyé } \\
\text { pyé a-y! } \\
\text { C'est le chagrin qui l'a mis là, } \\
\text { on voulait pas de lui par } \\
\text { rapport à son vieux pied! } \\
(130)\end{array}$ & $\begin{array}{l}\text { Comentarios } \\
\text { de la gente } \\
\text { sobre Léonce }\end{array}$ & Piedad & $\begin{array}{l}\text { Ø } \\
\text { La pena lo puso así, no le } \\
\text { quisieron por culpa de su pie } \\
\text { tullido (138) }\end{array}$ \\
\hline 12 & $\begin{array}{l}\text { Les nègres sans lendemain } \\
\text { (...) disent entre eux: Pli ou } \\
\text { an hangon, pli chyen ka } \\
\text { chiré-w. Célestina quant à } \\
\text { elle déclara quà toute chose } \\
\text { malheur est bon (169) }\end{array}$ & $\begin{array}{l}\text { La gente } \\
\text { desesperanz } \\
\text { ada según la } \\
\text { narradora, en } \\
\text { diálogo con } \\
\text { Celestina }\end{array}$ & Proverbio & $\begin{array}{l}\text { Los negros sin futuro (...) } \\
\text { dicen entre sí: "Cuantimás } \\
\text { pingos yevas, más te los } \\
\text { destriza el can." Celestina, } \\
\text { por su parte, declaró que no } \\
\text { hay mal que por bien no } \\
\text { venga... (176) }\end{array}$ \\
\hline 13 & $\begin{array}{l}\text { (...) Et kenbé rèd, pa moli! } \\
\ldots \text { (171) }\end{array}$ & $\begin{array}{l}\text { La narradora } \\
\text { a Celestina }\end{array}$ & Proverbio & $\begin{array}{l}-(\ldots) Y \text { guanta tiesa, no } \\
\text { tamolles... (179) }\end{array}$ \\
\hline 14 & $\begin{array}{l}\text { Ils marmottaient des "ki } \\
\text { moun é sa? Ola manzé } \\
\text { soti? Méyé sé on ti fanmi } \\
\text { Myrtha té ni granté! (174) }\end{array}$ & $\begin{array}{l}\text { La gente } \\
\text { sobre la } \\
\text { identidad de } \\
\text { la narradora }\end{array}$ & Chismorreo & $\begin{array}{l}\text { ¿Y esa quién é? ¿Daonde } \\
\text { salió señita? ¡Paíse ques } \\
\text { grande la familia de Myrtha! } \\
\text { (181) }\end{array}$ \\
\hline 15 & $\begin{array}{l}\text { le pa mélé mwen!... Ne me } \\
\text { mêle pas à ces histoires! } \\
(180)\end{array}$ & La narradora & Proverbio & $\begin{array}{l}\text { ¡No te mezcles en esa } \\
\text { historia! (188) }\end{array}$ \\
\hline 16 & $\begin{array}{l}\text { Alors, pa mélé mwen! ... } \\
\text { (180) }\end{array}$ & La narradora & Proverbio & $\begin{array}{l}\text { De modo que no-te- } \\
\text { meta...(188) }\end{array}$ \\
\hline 17 & $\begin{array}{l}\text { Lajan an ka aché, lanjan an } \\
\text { ka touvé! (183) }\end{array}$ & $\begin{array}{l}\text { Paul, gemelo } \\
\text { de Léonce, a } \\
\text { su tío Héctor }\end{array}$ & Consejo & $\begin{array}{l}\text { ¡La platita búcala, la platita } \\
\text { cuéntrala! (190) }\end{array}$ \\
\hline 18 & $\begin{array}{l}\text { Mwen enmé-w-manman! } \\
\text { (191) }\end{array}$ & $\begin{array}{l}\text { Dichos a } \\
\text { Mona } \\
\text { durante su } \\
\text { embriaguez }\end{array}$ & Seducción & ¡Ti-quero mama! (199) \\
\hline
\end{tabular}

Fuente: Elaboración propia

\section{Metodología y análisis de la inserción de oraciones en creole}

Para analizar la manera en que la autora inserta frases en creole en su escritura adaptamos la clasificación que propone Desmet Argain (307) para describir la amplia gama de mecanismos utilizados por el escritor para la inserción de oraciones en creole: 
1. Yuxtaposición de la traducción (con o sin marca; literal o libre);

2. Traducción con nota al pie;

3. Metalenguaje (el autor explica por qué el personaje dice lo que dice);

4. Traducción parcial;

5. Ausencia de traducción.

De estas opciones sólo encontramos en La grande drive des esprits la primera, la yuxtaposición, y la quinta, la ausencia de traducción. $Y$ debimos agregar una nueva categoría, la

\section{Traducción desplazada.}

Esta modalidad consiste en agregar la traducción no inmediatamente después de la inserción en creole sino diferida, intercalando otros segmentos entre el creole y su traducción. Este mecanismo puede considerarse como una herramienta de resistencia en donde la lengua minorizada representa un desafío a resolver por el lector no creolófono ante un efecto de alternancia de lenguas.

Entre estas variantes la escritora guadalupeña muestra una marcada tendencia a practicar la quinta modalidad, la de no acompañar las inserciones en creole de su traducción. Debemos destacar en este punto que no se observaron en La grande drive las modalidades de traducciones con notas al pie, ni las de metalenguaje que añaden explicación a los enunciados en creole, como tampoco se detectaron traducciones parciales. De los 18 ejemplos encontrados (tabla 2), 14 se insertan sin traducción, 3 van seguidos de su traducción y 1 es una traducción desplazada, como indicamos en la siguiente tabla.

\section{Tabla 3}

Clasificación de los ejemplos de la tabla 2 según los tipos de inserción de oraciones en creole en La grande drive des esprits.

\begin{tabular}{|l|l|l|}
\hline Tipos de inserción en el T.O... & Ejemplos & Total \\
\hline Ausencia de traducción & $1,3,5,6,7,8,9,10,12,13,14,16,17,18$ & $\mathbf{1 4}$ \\
\hline Yuxtaposición de la traducción & $2,11,15$ & $\mathbf{3}$ \\
\hline Traducción desplazada & 4 & $\mathbf{1}$ \\
\hline
\end{tabular}

Fuente: Elaboración propia

Comentaremos a continuación un ejemplo de cada categoría (tabla 3). Los ejemplos restantes los analizaremos en la siguiente sección junto con su traducción. En cada citación se indica en negrita el segmento en creole y al final, el número de página.

a. Ausencia de traducción. La omisión total de la traducción es la modalidad más frecuente de la autora en la que se basa para escribir en un continuo creole/francés, alternando una y otra lengua sin ninguna marca. Observemos el ejemplo 5 en que Man Octavie, la abuela ya fallecida de Léonce, se le aparece y le advierte:

\footnotetext{
${ }^{5}$ Con la sigla T.O.H., texto original heterolingüe, designamos al texto de partida, La grande drive des esprits.
} 


\section{Ejemplo $n^{\circ} 5$}

Zot ké ni kat ti moun! N'en demande pas davantage au Seigneur! (81)

Para acceder al sentido consultamos el diccionario de Raphael Confiant. Según esta fuente, a partir del pronombre personal zot, en francés vous, ustedes; la partícula ké que indica el futuro; $n i$, el verbo tener; kat, en francés quatre, el numeral cuatro; y ti moun que podría interpretarse como "niños"; la frase da la idea de que Octavie le está prediciendo a su nieto Léonce que tendrá cuatro hijos. Por ello podemos afirmar que se omite la traducción y lo que sigue es una consecuencia de lo dicho anteriormente.

b. Yuxtaposición de la traducción. En el ejemplo 11 la autora yuxtapone a la frase en creole su traducción literal sin ninguna marca, ni tipográfica ni léxica. Conserva la exclamación en estilo directo acerca de los comentarios que hacían los vecinos sobre Léonce:

\section{Ejemplo $n^{\circ} 11$}

Lapenn ki mété-y la! Lapenn pwan-y davwa yo pa té vlé-y, pa rapot a vyé pyé a-y! C'est le chagrin qui l'a mis là, on voulait pas de lui par rapport à son vieux pied! (130)

La narradora trae en discurso directo insertado en su narración las palabras de los vecinos que comentan los motivos por los cuales Léonce, sumido en el dolor empleaba toda su energía para trabajar la tierra. El no haber sido aceptado para unirse a la armada francesa para luchar contra la ocupación alemana en Francia, debido a su pie deformado, fue la causa de ese profundo dolor.

c. Traducción desplazada. La podemos observar en el ejemplo 4, donde Ninette, enojada con su hijo Léonce, le advierte de que deje de cuidar a su malvada abuela, Man Octavie:

\section{Ejemplo $n^{\circ} 4$}

Kitèy mò! Elle va te tuer, cher! Madanm-lasa sé on vyé volan! Laisse-la mourir! (76)

Este ejemplo muestra una estructura de alternancia de oraciones en las dos lenguas, en un continuo bilingüe: creole + francés + creole + francés. Para acceder al sentido de la primera expresión en creole, "Kitèy mò!", de nuestras búsquedas en el diccionario creole/francés de Confiant obtuvimos: CR Kité, FR Laisser, ES Dejar; y CR Mò, FR Mourir, ES Morir. A nuestro entender, esta expresión aparece traducida al francés en la última exclamación "Laisse-la mourir!" (ES ¡Déjala morir!) A su vez, la siguiente oración en francés "Elle va te tuer, cher!" (ES ¡Va a matarte, querido!), traduce no la idea que expresa la inserción en creole "Madanm-lasa sé on vyé volan!", sino más bien su efecto. En creole, vyé (o vié), en francés vieux (Confiant 1365), en español, viejo, es un adjetivo peyorativo, y el sustantivo volan, en francés volant, evoca a una persona que vuela gracias a poderes mágicos con intenciones de producir algún daño (Confiant 1374). Estos dos elementos, que forman parte de las creencias en lo sobrenatural de la 
cultura creole, desaparecen en la traducción en francés. Es preciso recordar que lo que hacemos aquí es describir los fenómenos lingüísticos observados, y las traducciones que el autor hace del creole constituyen recursos literarios dentro de su obra y no de un ejercicio de traducción profesional.

\section{Metodología y análisis de la traducción al español de oraciones en creole}

Nos dedicaremos ahora, luego de haber observado las diversas modalidades adoptadas por la autora para insertar sus oraciones en creole, al análisis de las opciones traductivas que tuvieron lugar para trasladar el heterolingüismo del texto fuente a la versión en español. Desde el punto de vista teórico, para llevar adelante nuestro estudio nos apoyamos en las perspectivas que se focalizan en el vínculo entre traducción e ideología. En lo que Bassnett llama etapa posestructuralista de los Estudios de Traducción, en la década de los noventa, toma protagonismo el debate sobre cuestiones como la primacía de la traducción frente al original, la diferencia y la periferia frente a los centros canónicos. Esta corriente, en términos de Calzada Pérez (94), concede vital importancia a la "nueva ética traductora" puesto que en el mundo posmoderno, bajo la influencia del posestructuralismo y el desconstructivismo, comienza una crítica hacia los fundamentos de la filosofía occidental (Tymoczko "Postcolonial Writing", "Translation of Themselves"; Vidal Claramonte; Meylaerts; Buzelin).

Desde el punto de vista metodológico, comparamos nuestras unidades de análisis, los binomios textuales (Rabadán; Alvarez Lugris) del texto heterolingüe original (T.H.O) y del texto heterolingüe traducido (T.H.T.), y los clasificamos según el grado de conservación del heterolingüismo. Para ello nos basamos en la nomenclatura propuesta por Javier Franco Aixelá para la traducción de elementos culturales la que rediseñamos en un trabajo previo (Ghirimoldi 119) para ajustarla a los objetivos planteados del presente trabajo. Aunque el autor de Culture-specific items in translation (Franco Aixelà 61-65) analiza la traducción de culturemas y ordena dichas técnicas según se orienten a la conservación de la referencia original o a la sustitución por elementos que se acercan a la lengua-cultura meta, nos vale como punto de referencia para nuestros análisis de conservación o eliminación del heterolingüismo.

Recordaremos cuáles son las técnicas de Franco Aixelá ordenadas del mayor al menor grado de manipulación, en su caso, intercultural, es decir, de la mayor a menor conservación de la referencia original por otra en la cultura receptora: repetición, adaptación ortográfica, traducción lingüística, glosa extratextual, glosa intratextual, sinonimia universalización limitada, universalización absoluta, naturalización, supresión y creación autónoma. Ahora describiremos cada una de estas técnicas, que adaptamos a nuestro análisis de elementos heterolingües. Reemplazamos la técnica de creación autónoma (que define el momento en que se agregan referencias culturales que no existen en el texto original) por la que denominamos "recreación lingüística". Esta recreación consiste en reemplazar el elemento heterolingüe por un español reinventado o creolizado que pretende trasponer características del creole en la lengua meta. Esta técnica se posiciona en el lugar de menor conservación del heterolingüismo

A continuación definimos las técnicas empleadas para nuestro análisis. Los ejemplos se presentarán de la siguiente manera: en la primera línea se muestra, en cursiva y entre comillas, el ejemplo del texto fuente en francés-creole, el texto 
heterolingüe original (T.H.O.) donde señalamos en negrita el segmento en creole; en la segunda línea se muestra la traducción publicada al español, el texto heterolingüe traducido (T.H.T.), precedida por el guión que indica en la obra traducida el discurso directo, inexistente en el texto de partida, indicando también en negrita el segmento en creole. Al final de ambos ejemplos se indica el número de página entre paréntesis.

\section{A. Técnicas de conservación del heterolingüismo}

En nuestro análisis sólo se pudo registrar la estrategia de repetición, como mostramos a continuación.

\section{A. 1. Repetición: conservación del creole}

Las inserciones en creole fueron conservadas en la traducción en sólo 3 casos de los 18 consignados. En el ejemplo 2 se conserva la oración en creole, sin marca, y sigue la traducción que hace la narradora en el texto original en francés.

\section{Ejemplo $\mathrm{n}^{\circ} \mathbf{2}$}

T.H.O. "On ti pisa an kay fè, chè! Pisser, seulement pisser, Ninette! » (61)

T.H.T. -On ti pisa an kay fè, chè! ¡Mear, sólo mear, Ninette! (66)

Se trata de una explicación teñida de fastidio que le da Sosthène a su mujer Ninette sobre los motivos por los cuales él se levanta de la cama por la noche. En la traducción del original se puede observar, por un lado, una transposición, en el cambio de categoría gramatical del sustantivo creole pisa, en francés pissat, urine (Confiant 1089), al verbo español "mear" de registro coloquial o malsonante como indica el diccionario de la RAE, eliminándose en consecuencia el adjetivo creole ti, en francés petit (Confiant 297), pequeño. En la traducción al español, se repite la misma estructura, es decir, se conserva la frase en creole y sigue su traducción como en el original.

En el ejemplo siguiente, observado más arriba, se combinan dos tipos de inserciones en creole, una traducción desplazada y una inserción sin traducción en un continuo creole-francés, y esta estructura es calcada en la versión al español con conservación del creole.

\section{Ejemplo $n^{\circ} 4$}

T.H.O. "Kitèy mò! Elle va te tuer, cher! Madanm-lasa sé on vyé volan! laisse-la mourir! » (76)

T.H.T. -Kitèy mò! ¡Va a matarte, querido! Madanm-lasa sé on vyé volan! ¡Déjala morir! (82)

A continuación, en el ejemplo 5, también comentado anteriormente, podemos observar cómo se conserva la frase en creole pero ahora sin ninguna traducción en el original, en un continuo de oraciones donde la autora pasa de una lengua a otra, estrategia que se conserva en la traducción:

\section{Ejemplo $n^{\circ} 5$}


T.H.O. "Zot ké ni kat ti moun! N'en demande pas davantage au Seigneur! » (81)

T. T. H. - Zot ké ni kat ti moun! ¡No pidas más al Señor! (88)

La frase en creole que emite Octavie, la abuela difunta de Léonce, para predecir a su nieto que va a tener cuatro hijos, no se traduce, y va seguida de otra oración en francés I español (en francés en el original y en español en la traducción) que continua con el discurso sobre la idea de no pedir más hijos a Dios.

\section{B. Técnicas de sustitución del heterolingüismo}

Nos ocupamos ahora de describir las técnicas detectadas durante el análisis de la traducción al español que tendieron a la sustitución del heterolingüismo: la universalización absoluta, la supresión y la recreación lingüistica, que se muestran a continuación.

\section{B. 1. Universalización absoluta: eliminación del creole y estandarización}

Dentro de la tendencia mayoritaria a suprimir el creole en los T.H.T, 1 caso corresponde a la traducción en español estándar, es decir, se traduce directamente del creole al español neutro. La técnica de traducción utilizada aquí es la universalización absoluta como la definimos de acuerdo con la clasificación de Franco Aixelá, en la que se elimina el elemento heterolingüe por un elemento neutro.

Ejemplo $\mathrm{n}^{\circ} 8$

T.H.O. «Bon dié» (97)

T.H.T. -Dios mío (104)

Esta expresión es insertada en la frase "Dis-moi Bon dié, dis-moi, ce que tu fais de moi!", traducida por "iDime, Dios mío, qué has hecho conmigo!", pronunciada por Sosthène en momentos de desesperación por haber perdido su fuerza viril, por lo cual se refugia en la lectura de la Biblia y en las plegarias. En la traducción se pierde toda huella del creole del original heterolingüe, neutralizado por un español estándar.

\section{B. 2. Supresión: eliminación total del creole}

Se encontró una ocurrencia en que, en el texto fuente, se hace uso del creole seguido de su traducción en francés; y en el texto traducido, se omitien totalmente las frases en creole, no quedando huella de su presencia.

Ejemplo $n^{\circ} 11$

T.H.O. « Lapenn ki mété-y la! Lapenn pwan-y davwa yo pa té vlé-y, pa rapot a vyé pyé a-y! C'est le chagrin qui l'a mis là, on voulait pas de lui par rapport à son vieux pied! » (130)

T.H.T. La pena lo puso así, no le quisieron por culpa de su pie tullido (138)

Como indicamos en la sección anterior sobre este ejemplo, se trata de una cita que hace la narradora para traer las voces de aquellos que comentaban las causas del 
dolor que sufría Léonce al no poder entrar en el ejército francés por su defecto físico. El creole aquí, la lengua hablada por la gente del pueblo, no se reproduce en la traducción.

\section{B. 3. Recreación lingüística: eliminación del creole y recreación del español}

En la obra traducida predomina la tendencia a eliminar el creole y recrear la traducción con una lengua meta deformada que reproduce la oralidad de un lenguaje coloquial familiar, como se observa en los ejemplos $1,3,6,7,9,10,12,13,14,15,16,17$ y 18. Tenemos la impresión de oír un lenguaje con cambios en la pronunciación y palabras acortadas, de sintaxis simplificada, que refleja un registro muy coloquial o de quien no domina un uso correcto de la lengua. Nos será de utilidad el concepto de "español creolizado", que utiliza Suchet al caracterizar el heterolingüismo textual de Juan Goytisolo en Juan sin tierra (1975), donde el poeta, para dar voz a las mujeres esclavas cubanas, reproduce fonéticamente el español, "contaminando el paradigma castellano" (Suchet 112), como en el siguiente ejemplo citado por la investigadora, donde unas negras dicen sobre una recién llegada: " (...) que no hay Dio que laclare que nosotra somo meno queya y que va adentá empatando con un cabronaso de blanco por mi madre bendita que ni pa calbón-calbón silve..." (ibid., 112).

De modo similar, en la novela de Pineau, se recrea la lengua meta, que podríamos llamar también español creolizado, como se observa en los siguientes 12 ejemplos relevados.

Ejemplo $\mathrm{n}^{0} 1$

T.H.O. « C'est bon! Ça peut aller! I ké kenbé! Ça va tenir!» (25)

T.H.T. ¡Ya está!, ¡puede servir!, ¡v'aguantar!, ¡resistirá! (28)

En el original aparece esta frase entrecomillada insertada en el relato de la narradora haciendo alusión a las palabras de aliento que los vecinos le dirigían a Léonce durante la construcción de su casa que aunque fuese precaria podría ser resistente. En la traducción al español el creole se borra y se traduce directamente al español creolizado mediante una aglutinación. Recordemos que en creole la partícula Ké ubicada delante del verbo forma el tiempo futuro (Pinalie y Bernabé 77).

Ejemplo $n^{\circ} 3$

T.H.O. "Lè pitit an mwen/ Ka mandé tété/ Mwen ka lé bay/ Manjé matété/ Pitit dodo/ Papa pa la/ Sé manman tou sèl/ Ki dan lanbara/ Pitit dodo/ Papa pa la/ Sémanman tou sèl/ Ki dan lanmizè...» (70)

T.H.T. Mi nenito/ Tere teta/ Ke le vi a da/ Pa komel puré/ Arroró/ Papa no ta/ Su mami ta sola/ No tiene na/ Arroró/ Papa no ta/ Su mami ta sola/ No tienún reá... (75)

El ejemplo 3 nos ofrece una canción de cuna escrita en su totalidad en creole y sin traducción ni explicación en francés en el texto de partida. En la obra traducida, aparece en un español recreado que reproduce el habla infantil, como en el empleo de "tere" en lugar de quiere. 


\section{Ejemplo $n^{\circ} 6$}

T.H.O. « -On bèl ti fi, bien belle, oui! » (89)

T.H.T. -¡Una bonita nena, mu bonita, sí! (96)

El ejemplo 6 muestra una parte del diálogo que tiene Ninette con su hijo Léonce cuando al descubrirlo tirado en el suelo luego de una borrachera, lo anima a que se reponga. En la traducción se repone el sentido en un español estándar con una leve recreación en el adverbio bien transformado en la palabra "muy" truncada en "mu". Aquí la recreación lingüística no se aplicó directamente al creole sino que se desplazó al segmento siguiente en francés que repite y refuerza la idea expresada en creole.

Ejemplo $n^{\circ} 7$

T.H.O. «Padon bondyé!» (91)

T.H.T. - ¡Peldón dio-mio! (98)

En esta ocasión la expresión en creole es emitida por la difunta Octavie dirigida a su nieto Léonce, durante la segunda oportunidad en que se le aparece, mientras éste se arrepiente de su borrachera y le pide perdón a Dios. Entonces su abuela irrumpe repitiendo estas palabras de Léonce, pronunciadas por él en un francés estándar pero repetidas por ella en creole. En la traducción al español se reemplaza el creole por una lengua recreada de registro más bajo con una pronunciación deformada.

Ejemplo $n^{\circ} 9$

T.H.O. "Sé ti moun! Yo pas biswen a yen dot ki manjé, kaka, pisé, domi! ...» (98)

T.H.T. ¡To chavale! ¡Tos comé, cagé, meá, dolmí! (105)

Se trata en el ejemplo 9 de las palabas de Agathe, la mujer india de Héctor, dirigidas a sus suegra Ninette, para que se quede tranquila por la salud de sus nietos.

Ejemplo $n^{\circ} 10$

T.H.O. « Bon dié, ban mwen kouraj! » (107)

T.H.T. -¡Dame való, dio mío! (113)

Estas palabras corresponden a la plegaria de Ninette pidiéndole a Dios que le de fuerza. Era el momento en que impulsada por las palabras de su hijo que insistía sobre los poderes de curación que tenía Ninette en sus manos, decide emplear ese don para ayudar a Sosthène, su marido que se encontraba abatido y abandonado.

\section{Ejemplo $n^{0} 14$}

T.H.O. "Ils marmottaient des "ki moun é sa? Ola manzé soti? Méyé sé on ti fanmi Myrtha té ni granté! » (174)

T.H.T. Murmuraban algunos "¿Y esa quién é? ¿Daonde salió señita? ¡Paíse ques grande la familia de Myrtha! (181) 
En este ejemplo se transcriben los comentarios que hacen los conocidos de la familia que van al entierro de Myrtha, quienes, al ver a la narradora llorando, se preguntaban quién era ella. En el español recreado de la traducción se pueden observar fenómenos de aglutinación y cambios de ortografía.

\section{Ejemplo $\mathrm{n}^{\circ} 15$}

T.H.O. "Et les épaules couvaient l'œuf de la lâcheté qui mûrit dans le pa mélé mwen!... Ne me mêle pas à ces histoires! » (180)

T.H.T. Y los hombros incubaban el huevo de cobardía que iba madurando en el no-te-meta... ¡No te mezcles en esa historia! (188)

Esta inserción pertenece a una escena relatada por la narradora en que, ante la violencia ejercida por un hombre sobre una mujer dentro de un bar, los clientes que asisten al hecho no reaccionan y prefieren cobardemente no intervenir. En la traducción al español, aunque se conserva el sentido, se borra el heterolingüismo del original y se pierde toda huella del creole. El efecto es de cierta redundancia pues se repite la misma información, una la del español recreado con la composición "no-te-meta" que traduce el creole del original, seguida de su equivalente "no te mezcles en esa historia", que es traducción de la traducción del creole en francés del original.

\section{Ejemplo $\mathrm{n}^{\circ} 16$}

T.H.O. « Alors, pa mélé mwen! ... » (180)

T.H.T. De modo que no-te-meta... (188)

Dentro del mismo relato del ejemplo anterior, aparece nuevamente esta expresión en creole en el original y sin traducción. En la versión española se muestra directamente la misma expresión recreada de "no-te-meta" que repite lo que ya había sido dicho unas frases antes.

\section{Ejemplo $n^{0} 17$}

T.H.O. « Lajan an ka aché, lanjan an ka touvé! » (183)

T.H.T -¡La platita búcala, la platita cuéntrala! (190)

La inserción del ejemplo 17 sale de la boca de Paul, uno de los hijos gemelos de Léonce, en respuesta a la pregunta de su tío Héctor, quien le pregunta si había encontrado la sabiduría, y su sobrino le contesta que no buscó a los sabios sino al dinero. El creole del original es transformado en un español con cambios ortográficos que reflejan una pronunciación incorrecta.

\section{Ejemplo $\mathrm{n}^{\circ} 18$}

T.H.O. « Mwen enmé-w-manman! » (191)

T.H.T. - iTi-quero mama! (199)

La frase en este ejemplo se le atribuye a Emmanuel dirigida a su madre Mona, bajo los efectos del ron. Era hijo de Sosthène y nació el mismo día en que su padre se casaba con Ninette. Mona, enferma de odio y venganza, maldijo al primer hijo de Sosthène, 
Léonce, y a toda su descendencia. La traducción al español borra el creole y recrea el español.

En último término comentaremos los ejemplos 12 y 13 de la tabla 2 que corresponden a proverbios en creole que aparecen en el texto fuente sin traducción dentro de un continuo francés-creole. En la traducción se apela a un español recreado que conserva el sentido literal del original creole.

\section{Ejemplo $n^{\circ} 12$}

T.H.O. "Les nègres sans lendemain (...) disent entre eux: Pli ou an hangon, pli chyen ka chiré-w. Célestina quant à elle déclara qu'à toute chose malheur est bon » (169)

T.H.T. Los negros sin futuro (...) dicen entre sí: "Cuantimás pingos yevas, más te los destriza el can" Celestina, por su parte, declaró que no hay mal que por bien no venga... (176)

El proverbio en creole del ejemplo 12 pertenece al relato de la serie de desgracias que caen sobre Léonce, cuando en el mismo año se convierte en huérfano y viudo. Esto sucede en el capítulo "Sabias palabras" que comienza con la frase: "Los filósofos dicen: las desgracias nunca llegan solas", que introduce la idea evocada en el proverbio.

La expresión pertenece a un dicho creole, que según el diccionario de Confiant (282), en la entrada chien, significa:

Pli ou chiré, pli chien raléw. (Lit. Plus on est en haillons, plus le chien vous mord aux mollets): Le Malheur appelle le Malheur.

Si traducimos literalmente la explicación en francés del diccionario de Confiant, obtendríamos una frase del tipo: "Más harapos llevamos puestos, más nos muerde el perro en los tobillos". Como podemos ver, la opción del traductor fue calcar el proverbio creole en la lengua meta recreada en lugar de optar por la naturalización y reemplazarlo por otro proverbio español para la desgracia, del tipo "A perro flaco todos son pulgas". A pesar de borrar en la traducción el heterolingüismo del original, se conserva el concepto que proviene del imaginario criollo.

El siguiente proverbio muestra las palabras pronunciadas por la narradora a Celestina cuando le anuncia la muerte de su madre, Myrtha, la esposa de Léonce, para darle ánimo en ese momento doloroso.

Ejemplo $n^{\circ} 13$

T.H.O. «Et kenbé rèd, pa moli! ... » (171)

T.H.T. -Y guanta tiesa, no tamolles... (179)

Según el diccionario de Confiant, rèd es duro, difícil, firme y moli ceder, bajar los brazos. Encontramos en el Petit Dictionnaire Insolite des Cultures et des Langues créoles de Suzy Palatin, que estamos aquí ante una expresión creole "kinbé rèd", en francés Tiens bon, que corresponde a palabras de aliento. La frase "Kimbé rèd pa moli sé moli la ki rèd", significa en francés Tiens bon, ne faiblis pas, c'est mollir le plus difficile (Palatin 62). En español sería algo así como: "ten valor, no te desanimes". Con 
la misma expresión comienza una canción de Guy Conquête, cantante y músico guadalupeño: "Kimé rèd, fwèr kimbé rèd surtou pa moli /Douvan misié la ka kimbé fwèt la" que significa "Aguanta, muéstrate firme hermano, sobre todo no cedas / Frente al hombre que tiene el látigo" (ibid.62).

En la traducción, la recreación lingüística conserva el sentido. De acuerdo a nuestras consultas en el diccionario de la RAE, encontramos como entrada el verbo "amollar", de muelle, que entre otros significados quiere decir "ceder, aflojar, desistir". La recreación "tamolles" sería una lexicalización donde dos unidades (te + amolles) se fusionan en una palabra compuesta lexicalizada.

\section{Discusión: Elogio de la marginalidad por parte del traductor}

En esta última etapa reflexionaremos sobre los resultados obtenidos con el fin de elaborar algunas conclusiones, provisorias, acerca de la tendencia mostrada a través de las decisiones del traductor a conservar el heterolingüismo, es decir, elogiar la creolidad, o por el contrario, a sustituirlo, es decir, elogiar la marginalidad de la lengua minorizada.

Si repasamos el proyecto estético que se propone la autora con la novela, el de mostrarse al mundo como una autora guadalupeña a pesar de haber nacido en París, e impregnar su escritura con el creole, es lícito preguntarse si en la traducción sobreviven las marcas suficientes como para que el lector esté en condiciones de percibir esa intención fundadora, percibir esa matriz creole que surge del interior de la poeta que da voz a la narradora y a otros personajes que no podrían expresarse de otro modo en determinadas circunstancias.

De lo observado en nuestros ejemplos se desprende la imagen de un traductor activo y recreador que toma la decisión de borrar el heterolingüismo en la mayoría de los casos (83\%), a través de la universalización absoluta, de la supresión o de la recreación lingüística, con lo cual el pacto de lectura bilingüe del original se ve seriamente comprometido. Estamos frente a un caso evidente de manipulación, en términos de Theo Hermans, donde el traductor introduce una variedad de español popular para sustituir la presencia del creole. Esta estrategia introduce un cambio significativo en la intención de Pineau de dar espacio a la lengua minorizada en la escritura, poniéndola en un nivel de igualdad con la lengua francesa. Al no ofrecer traducciones la autora lleva a cabo su resistencia contra el monolingüismo y la dominación eurocéntrica que exige un pacto de lectura bilingüe. Todo esto queda anulado y transformado por parte del traductor mediante la recreación de una variación dialectal que conlleva efectos contrarios a los deseados en el original, cargando de marginalidad a la lengua dominada.

De los ejemplos observados en los que el creole se reemplazó por un español recreado detectamos varios mecanismos que encuentran puntos en común con rasgos característicos del habla andaluza o español meridional, una rama del castellano que se difundió especialmente en el área del Caribe (Gámez Millán y Agón Ariza 128). Este último detalle podría estar relacionado con la decisión del traductor de establecer un vínculo entre esta variedad y el territorio caribeño. Entre estos rasgos encontramos los siguientes: 
1. elisión de consonantes finales: se eliminan las " $r$ " finales, por ejemplo: "da" por" dar"; se eliminan las "n" finales: "comé" por "comen";

2. sustitución de /r/ por /l/: "komel" por "comer";

3. pérdida de consonantes en mitad de palabra: "búcala" en lugar de "búscala";

4. yeísmo: pronunciación de la elle /ll/ como ye /y/: "yevas" por "llevas";

5. truncamiento de palabras por pérdida de una sílaba: aféresis para la forma "ta" por "está"; "to" por "estos";

6. pérdida de la sílaba final: "pa" en lugar de "para";

7. alargamiento de las vocales ante eliminación de la consonante o vocal final: "dio" por "dios"; "mu" por "muy;

8. aglutinación y lexicalización: cuando dos elementos se fusionan y crear una nueva palabra como en "v'aguantar" por "va a aguantar"; "tienún" por "no tiene ni un"; "señita" por "señorita"; "daonde" por "de dónde";

Además de estas características que emparentan la recreación que lleva a cabo el traductor con el habla andaluza, comentaremos otra técnica empleada en la traducción al español de algunos elementos presentes en las oraciones insertadas: la naturalización, es decir, la sustitución del elemento heterolingüe por un elemento específico de la lengua meta (siguiendo la nomenclatura de Franco Aixelá para la traducción de culturemas). Mostramos aquí algunos ejemplos:

1. "matété" en creole, un plato a base de harina de mandioca y de leche (Confiant, 2007, p. 954), que es reemplazado por "puré";

2. "lanmizé", en creole, la miseria, aquí la pobreza de la madre, es recreada con la locución coloquial "no tienún reá" ( no tiene ni un real);

3. "ti moun", niños en creole, es reemplazado por "chavale", préstamo del caló, niños o muchachos;

4. "ki dan lanbara", expresión creole que significa "que está en dificultades" (del francés "qui est dans l'embarras", Confiant 790), es traducida por la expresión "no tiene na".

Ahora bien, se podría argumentar por otra parte que en el español reinventado en la traducción es posible percibir la presencia subyacente del creole, la lengua eliminada en la traducción. Decimos esto basándonos en los rasgos observados en la lengua recreada que son comunes al creole, y que agrupamos en dos categorías: 1. alteración de la grafía y, 2. la aglutinación.

1. Alteración de la grafía que refleja la pronunciación:

a. En creole, no existe la letra "c", toda palabra que lleve el sonido /k/ se escribe con la letra "K": CR ${ }^{6}$ kristal, ES cristal; CR kontinent, ES continente; CR kafé, ES café; etc. En el español recreado, como dijimos más arriba, se observó el cambio de "komel" por "comer", o de "ke" por "que" en "ke le vi a da" (ejemplo n 3 ).

b. En creole como en francés, las " $r$ " finales no se pronuncian, directamente no aparecen en la grafía, como por ejemplo el verbo en FR aller, ES ir, en CR es allé. En el español recreado, se observó el término "da" en lugar de "dar".

2. Aglutinación:

a. También es típico del creole la adjunción del artículo definido al sustantivo que se lexicalizó: CR lajwa, FR la joie, ES la alegría; o CR lajistis, FR la justice, ES la

${ }^{6}$ Utilizamos las siglas: FR, francés; CR, creole; ES, español. 
justicia. En el español recreado observamos, por ejemplo, la aglutinación y lexicalización de "v’aguantar" por "va a aguantar".

Estos mecanismos, que mostramos a título de ejemplo de transferencia de características del creole al español reinventado, constituyen uno de los modos en que puede percibirse la presencia del creole eliminado. $Y$ este hecho nos recuerda el concepto de Tymoczko (Translation in a Postcolonial Context) de traducción como reescritura metonímica, donde un atributo de la lengua que se transfiere en la traducción representa la lengua creole en su conjunto. Vale preguntarse (y sería fuente lícita de una posible investigación futura) si el lector de la obra en español, al encontrarse con ese registro de lengua, oye las resonancias de la oralidad y de la creatividad lingüística del creole. O por el contrario, percibe una lengua deformada, un basilecto, que remite a un pueblo marginado y sin educación. En este último caso, aunque se reciban ecos de la lengua subyacente en el original, el efecto en la recepción se aleja de la intención de la autora, la de revalorizar la lengua marginada. ¿No parece acaso una traducción re-colonizadora, que vuelve a someter el creole al yugo de la lengua dominante, y es llevada hasta su versión basilectal? Y aquí es donde no solo se pone en evidencia la manipulación que ejerce el traductor en su elección de las estrategias traductivas sino también se plantea, de manera más positiva, la traducción como espacio de creatividad e innovación. La traducción del heterolingüismo, como plantea Buzelin puede verse no tanto como un problema sino como una oportunidad para los Estudios de Traducción de promover enfoques eclécticos para comprender las funciones del plurilingüismo en la traducción así como conceptos flexibles y multifacéticos. Recordemos cuando Borges decía que no hay textos definitivos, éstos solo corresponden a la religión o al cansancio, solo hay borradores y versiones (Waisman 69). Pensemos entonces, junto con María Constanza Guzmán, en otras versiones que defiendan la traducción como praxis decolonial, como la que lleva adelante la revista cubana fundada en 1959 Casa de las Américas, que traduce el Caribe para América Latina, donde la práctica traductora responde a un proyecto intelectual y político antiimperialista, latinoamericanista, contrahegemónico y descolonizador (Guzmán 178). Y con esto abrimos nuevas puntos de reflexión que hacen evolucionar nuestra querida disciplina, la Traductología.

\section{Obras citadas}

Alvarez Lugris, A. "En torno a la unidad de traducción y la unidad de análisis de la traducción en estilística comparada." Hermeneus. Revista de Traducción e Interpretación no. 3, 2001, pp. 61-81.

Arsaye, J.-P. Français-Créole/Créole-Français. De la traduction. Ethique. Pratiques. Problèmes. Enjeux. L'Harmattan, 2004.

Ashcroft, B., G. Griffiths, y H. Tiffin. The Empires Writes Back. Theory and Practice in Post-colonial Literatures. Routledge, 1998. 
Bandia, P. "The Impact of Postmodern Discourse on the History of Translation." Charting the Future and Translation History, editores P. F. Bandia y G. L. Bastin, University of Ottawa Press, 2006, pp. 45-58

Bernabé, J. Approche cognitive du créole martiniquais. L'Harmattan, 2015.

Bernabé, J., P. Chamoiseau, y R. Confiant. Eloge de la créolité. Gallimard, 1993.

---. Elogio de la creolidad. Traductores M. M. Del Valle Idárraga, y Martin-Laprade, Editorial Pontificia Universidad Javeriana, 2011.

Buzelin, H. "Traduire I'hybridité littéraire: Réflexions à partir du roman de Samuel Selvon: The Lonely Londoners." Target vol. 18, no. 1, 2006, pp. 91-119.

Calzada Pérez, M. El espejo traductológico: teorías y didácticas para la formación del traductor. Octaedro, 2007.

Casanova, P. La República mundial de las Letras. Anagrama, 2001.

Confiant, R. Dictionnaire créole-francais. Ibis Rouge, 2007.

Deleuze, G., y F. Guattari. Kafka. Por una literatura menor. Ediciones Era, 1978.

Desmet Argain, C. El translingüismo en Texaco de Patrick Chamoiseau: Implicaciones en la traducción de textos híbridos. 2015. Universidad Nacional Autónoma de México, tesis de doctorado.

De Souza, P. "Inscription du créole dans les textes francophones: de la citation à la créolisation." Penser la créolité, editores M. Condé y Madelaine et CottenetHage, Karthala, 1995, pp. 173-190.

Franco Aixelá, J. "Culture-specific Items in Translation." Translation, Power, Subversion, editores R. Alvarez y M.C.A. Vidal Claramonte, Multilingual Matters, 1996, pp. 61-65.

Gámez Millán, A. F. y M. Agón Ariza. "Análisis de rasgos lingüisticos en el texto andaluz. La infancia de Jesu-Christo desde una perspectiva sociohistórica y cultural." Cuadernos de Lingüística Hispánica, no. 25, 2014, pp. 123-142.

Ghirimoldi, M. E. "La lengua mestiza de Patrick Chamoiseau en Texaco. Análisis de las Técnicas de su traducción al español." Mutatis Mutandis, vol. 10, no. 1, 2017, pp. 116-139.

Glissant, É. El Discurso Antillano. Casa de las Américas, 2010.

Grutman, R. Des langues qui résonnent. L'hétérolinguisme au XIX siècle québécois. Fides, 1997.

Guzmán, M. C. "El Caribe se traduce: la traducción como praxis descolonial en las revistas Tropiques, Bim y Casa de las Américas." Mutatis Mutandis, vol. 10, no. 1, 2017, pp. 167-181.

Hazael-Massieux, M. C. "Le créole dans le roman des années 1990 aux Antilles: de la réalité au mythe?/Creole in the French Caribbean Novel of the 1990s: From Reality to Myth?" The Francophone Caribbean Today. Literature, Language, Culture, editor G. Aub-Buscheer, University of the West Indies Press, 2003, pp. 82-101, creoles.free.fr/articles/roman 1990\%20aux\%20Antilles.pdf. Accedido 7 de feb 2020.

Hermans, T. The Manipulation of Literature. Studies in Literary Translation. Croom Helm, 1985.

Kundera, M. "Beau comme une rencontre multiple." L'infini, no. 34, 1991, pp. 50-62. 
Mehrez, S. "Translation and the Postcolonial Experience: The Francophone North African Text." Rethinking Translation: Discourse, Subjectivity, Ideology, editor L. Venuti, Routledge, 1992, pp. 120-138.

Meylaerts, R. "Heterolinguism in/and translation: How legitimate are the Other and his/her language?" Target, vol. 18, no. 1, 2006, pp. 1-15.

Palatin, S. Petit dictionnaire insolite des cultures et des langues créoles. Guadaloupe, Guyanne, Martinique. Larousse, 2013.

Pinalie P. y J. Bernabé. Grammaire du Créole Martiniquais. L'Harmattan, 1999.

Pineau, G. La grande drive des esprits. Editions Philippe Rey, 1993.

---. "Le Sens de mon écriture." LittéRéalité, vol. 10, no. 1, 1998, pp. 135-136.

---. Una antigua maldición. Traductor M. S. Crespo, Ediciones del bronce, 1999.

---. "L'identité, la créolité et la francité." La culture francaise vue d'ici et d'ailleurs, editor T. C. Spear, Karthala, 2002, pp. 217-224.

Rabadán, R. Equivalencia y traducción: Problemática de la equivalencia translémica inglés-español. Universidad de León, 1991.

Santoyo, J. C. y R. Rabadán. "Basic Spanish Terminology for Translation Studies: a Proposal." Meta, vol. 1, no. XXXVI, 1991, pp. 318-322.

Scott, P. "Gabriel Okara's The Voice: The non-ljo reader and the pragmatics of translinguism." Research in African Literatures, vol. 21, no. 3, 1990, pp. 75-88.

Suchet, M. Outils pour une traduction postcoloniale. Editions des archives contemporaines, 2009.

Tymoczko, M. "Post-colonial writing and literary translation." Post-Colonial Translation. Theory and Practice, editores S. Bassnett y H. Trivedi, Routledge, 1999: pp. 1940.

---. Translation in a Postcolonial Context. Early Irish Literature in English Translation. St. Jerome, 1999.

---. "Translation of Themselves: the Contours of Postcolonial Era." Changing the Terms: Translating in the Postcolonial Era, editores S. Simon y P. St-Pierre, University of Ottawa Press, 2000, pp. 147-163.

Vidal Claramonte, M. C. A. En los límites de la traducción. Comares, 2005.

Waisman, S. Borges y la traducción. Adriana Hidalgo editora, 2005.

Zabus, C. The African Palimpsest: Indigenization of Language in the West African Europhone Novel. Rodopi, 2004. 